D. W. PiotrowSKi,* A. S. KAMLET,* A.-M. R. DECHERT-SCHMitT, J. YAN, T. A. BRANDT, J. XIAO, L. WEI, M. T. BARRILA (PFIZER INC., GROTON, USA)

Regio- and Enantioselective Synthesis of Azole Hemiaminal Esters by Lewis Base Catalyzed Dynamic Kinetic Resolution

J. Am. Chem. Soc. 2016, 138, 4818-4823.

\title{
Lewis Base-Catalyzed Dynamic Kinetic Resolution of Azole Esters
}
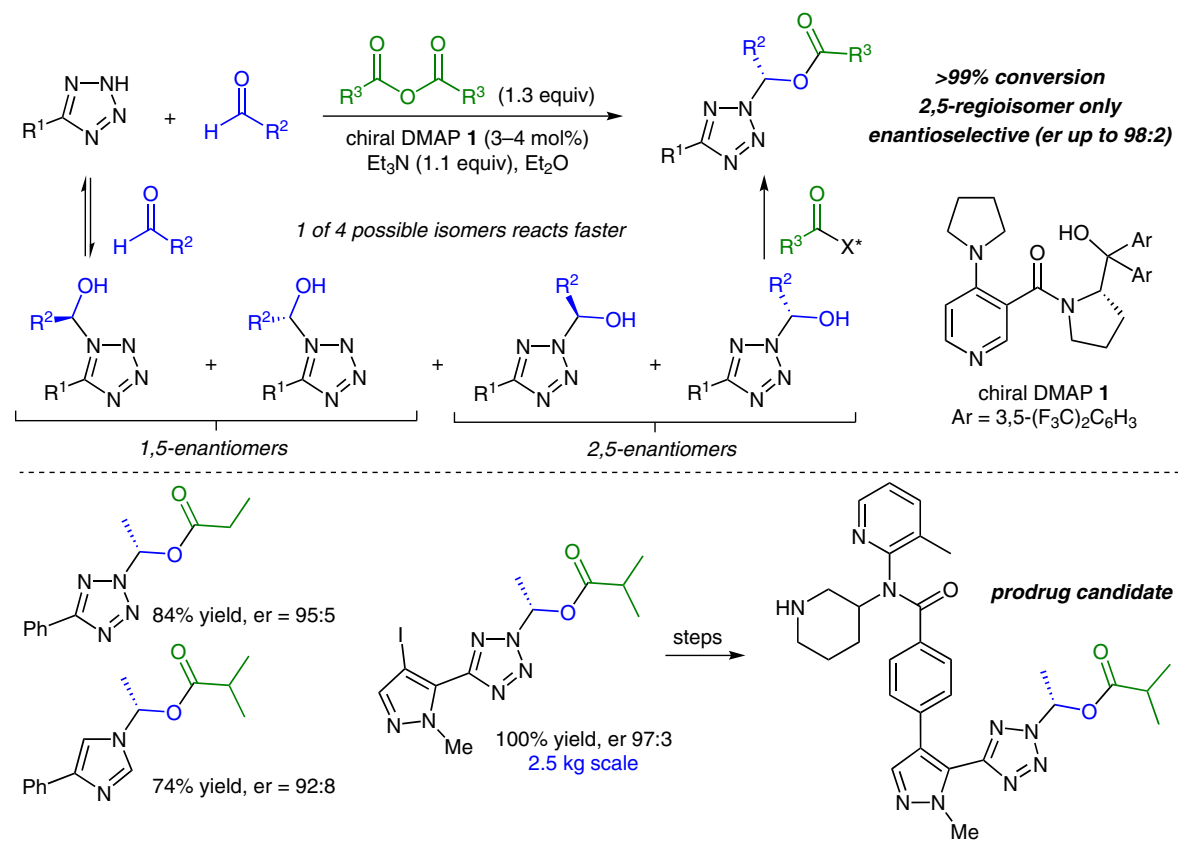

Significance: The tetrazole motif is a well-known carboxylic acid isostere and, as such, part of a valuable class of heterocycles in drug-discovery programs. Access to $\mathrm{N}$-substituted tetrazoles is typically plagued by poor regioselectivities (i.e., mixtures of 1,5- and 2,5-regioisomers) or harsh conditions. A mild and completely regioselective method is reported for the synthesis of 2,5-disubstituted tetrazoles with chiral $N$-hemiaminal esters. The products are formed by treating unsubstituted tetrazoles with aldehydes and anhydrides in the presence of triethylamine and a Lewis base (DMAP). The use of a prolinol-based chiral DMAP catalyst permitted dynamic kinetic resolution (DKR) to occur, generating 2,5-substituted tetrazoles in high enantiomeric ratios. This method was extended to other azoles (imidazoles, pyrazoles) and was applied to the large-scale $(2.5 \mathrm{~kg})$ synthesis of a prodrug candidate.

SYNFACTS Contributors: Victor Snieckus, Julien Genovino Synfacts 2016, 12(06), 0565 Published online: 17.05.2016 Dol: 10.1055/s-0035-1562223; Reg-No.: V05216SF
Comment: The method is a unique example of a regio- and enantioselective DKR for an entry into 2,5-substituted hemiaminal esters. The DKR takes advantage of the equilibrium between four transient hemiaminal adducts (see scheme). A kinetic analysis reveals that mixtures of hemiaminal adducts can be observed (NMR) for sterically hindered substrates. However, high regioselectivity and enantioselectivity can be achieved in the ratedetermining acylation step. Overall, the yields (71$100 \%)$, regioselectivity (100:0), and enantiomeric ratio (s98:2) are excellent. Several anhydrides, aliphatic aldehydes (linear, $\alpha$-branched), and benzaldehydes were used successfully, and the scope was also extended to hemiaminal carbonates and sulfonates. This method provides access to a range of enantioenriched azole prodrugs, so that their rates of enzymatic hydrolysis can be tailored to the needs of medicinal chemistry projects.
Key words

kinetic resolution

asymmetric synthesis

tetrazoles

hemiaminal esters

prodrugs

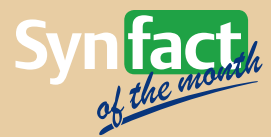

